\title{
An Invasive and a Noninvasive Approach for the Automatic Differentiation of Obstructive and Central Hypopneas
}

\author{
Christian Morgenstern*, Member, IEEE, Matthias Schwaibold, Winfried J. Randerath, Armin Bolz, \\ and Raimon Jané, Member, IEEE
}

\begin{abstract}
The automatic differentiation of obstructive and central respiratory events is a major challenge in the diagnosis of sleep-disordered breathing. Esophageal pressure (Pes) measurement is the gold-standard method to identify these events. This study presents a new classifier that automatically differentiates obstructive and central hypopneas with the Pes signal and a new approach for an automatic noninvasive classifier with nasal airflow. An overall of 28 patients underwent night polysomnography with Pes recording, and a total of $\mathbf{7 6 9}$ hypopneas were manually scored by human experts to create a gold-standard annotation set. Features were automatically extracted from the Pes signal to train and test the classifiers (discriminant analysis, support vector machines, and adaboost). After a significantly $(p<0.01)$ higher incidence of inspiratory flow limitation episodes in obstructive hypopneas was objectively, invasively assessed compared to central hypopneas, the feasibility of an automatic noninvasive classifier with features extracted from the airflow signal was demonstrated. The automatic invasive classifier achieved a mean sensitivity, specificity, and accuracy of 0.90 after a 100 -fold cross validation. The automatic noninvasive feasibility study obtained similar hypopnea differentiation results as a manual noninvasive classification algorithm. Hence, both systems seem promising for the automatic differentiation of obstructive and central hypopneas.
\end{abstract}

Index Terms-Automatic differentiation, central hypopnea, esophageal pressure (Pes), inspiratory flow limitation (IFL), noninvasive classification, obstructive hypopnea.

Manuscript received December 17, 2009; revised February 20, 2010; accepted March 25, 2010. Date of publication April 15, 2010; date of current version July 14,2010 . This work was supported in part by the Spanish Ministerio de Ciencia e Innovación under Grant TEC2007-68076-C02-01. Asterisk indicates corresponding author.

${ }^{*}$ C. Morgenstern is with the Institut de Bioenginyeria de Catalunya (IBEC), Departament d'Enginyeria de Sistemes, Automàtica i Informàtica Industrial (ESAII), Universitat Politècnica de Catalunya (UPC), and Centro de Investigación Biomédica en Red (CIBER) de Bioingeniería, Biomateriales y Nanomedicina (CIBER-BBN), E-08028 Barcelona, Spain (e-mail: cmorgenstern@ibec.pcb.ub.es).

M. Schwaibold is with the MCC GmbH \& Co. KG, D-76135 Karlsruhe, Germany (e-mail: matthias.schwaibold@mcc-med.de).

W. J. Randerath is with the Klinikum Bethanien, D-42699 Solingen, Germany, and also with the Institute for Pneumology, University of Witten/Herdecke, 58448 Witten, Germany (e-mail: randerath@klinik-bethanien.de).

A. Bolz is with the Institute of Biomedical Engineering, Karlsruhe Institute of Technology, D-76131 Karlsruhe, Germany (e-mail: armin.bolz@ibt. uni-karlsruhe.de).

R. Jané is with the Institut de Bioenginyeria de Catalunya (IBEC), Departament d'Enginyeria de Sistemes, Automàtica i Informàtica Industrial (ESAII), Universitat Politècnica de Catalunya (UPC), and El Centro de Investigación Biomédica en Red (CIBER) de Bioingeniería, Biomateriales y Nanomedicina (CIBER-BBN), E-08028 Barcelona, Spain (e-mail: rjane@ibec.pcb.ub.es).

Color versions of one or more of the figures in this paper are available online at http://ieeexplore.ieee.org.

Digital Object Identifier 10.1109/TBME.2010.2047505

\section{NOMENCLATURE}

CSAHS

DA

flow2min

hflow

$h P e s$

IFL

MA

NPSG

OSAHS

$(P / \dot{V})$ relationship

Pes

Pes2min

PSG

SD

SDB

UA
SVM

Central sleep apnea/hypopnea syndrome. Discriminant analysis.

Flow signal of the 2 min preceding a hypopnea's onset.

Flow signal of a hypopnea.

Pes signal of a hypopnea.

Inspiratory flow limitation.

Moving average.

Night polysomnography.

Obstructive sleep apnea/hypopnea syndrome.

Esophageal pressure/airflow-relationship.

Esophageal pressure.

Pes signal of the 2 min preceding a hypopnea's onset.

Polysomnography.

Standard deviation.

Sleep-disordered breathing.

Support vector machine.

Upper airway.

\section{INTRODUCTION}

$\mathbf{S}$ LEEP-DISORDERED breathing (SDB) comprises a broad array of disorders characterized by abnormalities of respiratory pattern and ventilation during sleep. One of the most important aspects for the appropriate choice of treatment in SDB is the correct identification of respiratory events. Particularly, the correct differentiation between central and obstructive apneas/hypopneas is one of the most recurrent tasks in clinical practice due to the high prevalence of the corresponding obstructive sleep apnea/hypopnea syndrome (OSAHS) and central sleep apnea/hypopnea syndrome (CSAHS) [1] and their diametrically different treatments [1], [2]. For decades, esophageal pressure (Pes) measurement has been the gold-standard technique for measuring respiratory effort [3], [4], as it is a much more sensitive technique than classical night polysomnography (NPSG) techniques that usually overestimate the incidence of central events [5]. Consequently, the assessment of variations in Pes swings is still the most reliable differentiation criterion for central and obstructive events [3], [4].

Nonetheless, the complexity and invasiveness of Pes manometry and its impact on sleep [6] limits its usage in clinical routine. Researchers have been recently trying to develop methods 
for the noninvasive differentiation of central and obstructive apneas/hypopneas with different approaches, such as wavelet analysis of the airflow signal [7], forced-oscillation technique (FOT) [8], [9], and pulse-transit-time (PTT) [10]. However, the clinical adoption of these techniques has been limited due to the difficultness to achieve validation in clinical routine. Goldstandard validation is usually achieved by comparing a system's scorings with an extensive, manually scored (Pes) annotation set. However, the manual scoring of the Pes signal represents a laborious task, as the manual identification of respiratory events by a human expert is a cumbersome procedure and may suffer from interscorer differences and subjective interpretation. Hence, the development of an objective and efficient method for the automatic invasive assessment of central and obstructive events is still an ongoing challenge in SDB research, as new invasive approaches are still being suggested [5].

In this paper, we propose, in a first part, a new automatic classifier [11] based upon supervised machine-learning methods that would automatically allow to differentiate central and obstructive hypopneas with the gold-standard Pes signal. This would permit the automatic and efficient creation of extensive annotation sets that could be used for the clinical validation of hypopnea differentiation systems. Our system focused exclusively on the differentiation of hypopneas, as the pressure swings during a hypopnea are more subtle than during other SDB events, therefore being considered the more challenging classification task. Nevertheless, the clinical severity and the pathological consequences of the obstructive sleep hypopnea syndrome (OSHS) are on par to those of the OSA syndrome (OSAS) [1], [12]. In a first step, 769 hypopneas were manually scored by human experts with the PSG and Pes signals, according to standard criteria [3] to create a gold-standard annotation set. Then, a specific set of features was automatically extracted from the Pes signal to characterize the two classes of hypopneas. These features were used to train and test our new automatic classifiers. Finally, the differentiation performance of each classifier was evaluated by comparing the automatic classification results with the manual, gold-standard Pes annotations.

However, as Pes measurement is an invasive technique that is not used in clinical routine, we propose in the second part of this paper, a new approach that should show the feasibility of a noninvasive automatic differentiation system of central and obstructive hypopneas. A characteristic airflow pattern (flattening) can be observed in inspirations with inspiratory flow limitation (IFL). IFL is usually caused by increased upper airway (UA) resistance that prevents the increase of airflow despite increasing respiratory effort [13]-[15]. As increased UA resistance is usually also the main cause of obstructive hypopneas [1], [3], [4], we hypothesized that obstructive hypopneas should present a significantly higher incidence of IFL episodes than central hypopneas. In order to prove this hypothesis, we used the invasive Pes-flow $(P / \dot{V})$ relationship [14]-[16] to objectively assess and compare the incidence of IFL in the inspirations of each hypopnea class. Nevertheless, recent studies [13], [16] have shown that IFL episodes can also be assessed noninvasively solely with the airflow signal. Thus, if the IFL-related information contained in a hypopnea should prove to be relevant for the automatic auto- matic differentiation process, it would be feasible to develop an automatic, entirely noninvasive hypopnea classification system solely based upon the airflow signal.

\section{Materials AND Methods}

\section{A. Subjects}

Twenty-eight subjects had full night PSG (NPSG) with an 18-channel recorder (Somnolab sleep diagnosis system, Weinmann GmbH, Hamburg, Germany) at the sleep laboratories of Klinikum Bethanien hospital in Solingen, Germany. The clinical protocol was specifically designed for these purposes and approved by the hospital's Ethics Committee. Twentythree subjects were male and five were female. Mean values $\pm \mathrm{SD}$ of the studied population for apnea-hypopnea index (AHI) were $18.9 \pm 18.5$ events/h (range 2.3-91.2 events/h) with a hypopnea index (HI) of $10.4 \pm 6.6$ events/h (range 1.1-27.3 events/h), body mass index (BMI) $28.5 \pm 4.5 \mathrm{~kg} / \mathrm{m}^{2}$ (range $21.0-41.9 \mathrm{~kg} / \mathrm{m}^{2}$ ), and age $52.6 \pm 15.6$ years (range $23-$ 78 years). Given the complexity of Pes recording, the number of patients in our study represents an up to a threefold increase in the overall cohort size in comparison to other studies with Pes measurement [10], [13]-[15].

\section{B. Data Acquisition}

To obtain the respiratory signal, a nasal cannula device (Weinmann $\mathrm{GmbH}$ ) was connected to a pressure transducer system (Weinmann $\mathrm{GmbH}$ ). These systems are indicated by current NPSG guidelines [3], [4] for the identification of hypopneas and have been routinely used with the Pes signal for the identification and analysis of IFL [13], [14], [16], [17]. The airflow signal was recorded with a sampling frequency of $32 \mathrm{~Hz}[4]$ and an 8-bit resolution.

Pes was recorded with an unidirectional pressure-tip catheter (UniTip catheter by UNISENSOR AG, Attikon, Switzerland) that consisted of a piezoresistive pressure sensor with an accuracy of $\pm 0.6 \mathrm{mmHg}$, a sensitivity of $5 \mu \mathrm{V} /(\mathrm{V} \cdot \mathrm{mmHg})$, and a typical resolution of $[-100, \ldots,+300 \mathrm{mmHg}]$. A separate pressure amplifier (ISOPRE-P amplifier, Standard instruments $\mathrm{GmbH}$, Karlsruhe, Germany) with a resolution of $[-99 \mathrm{mmHg}, \ldots$, +200 $\mathrm{mmHg}$ ] was connected to the catheter. The Pes signal was recorded with a sampling frequency of $16 \mathrm{~Hz}$ and a 12-bit resolution. The sensor was calibrated before each measurement by introducing the catheter in a sealed cylinder, by manually incrementing the pressure inside the cylinder with a pressure manometer from 0 to $100 \mathrm{mmHg}$ and manually setting the calibration markers in the pressure amplifier. The catheter was introduced through the patient's nostrils after spraying the nasopharynx with xylocaine and positioned in the lower third of the esophagus [18], [19].

Other physiological signals, like arterial oxygen saturation $\left(\mathrm{SpO}_{2}\right)$, body position, pulse, and thoracic and abdominal respiratory inductance plethysmography (ProTech, Services Inc, Mukilteo, WA, USA) were recorded with an 8-bit resolution. Also, two electroencephalogram channels (C3-A2/C4-A1), two electrooculogram (right/left), one submental electromyogram 
(EMG), one leg-EMG, and an ECG channel were systematically recorded with a sample frequency of $256 \mathrm{~Hz}$ and a 12-bit resolution, respectively.

\section{Event Annotations}

Sleep stages, apneas, hypopneas, and other respiratory events were scored by applying standard criteria [3]. According to these criteria, a hypopnea lasts for at least $10 \mathrm{~s}$ and is identified by a clear decrease $(>50 \%)$ in the amplitude of a valid measure of breathing from baseline during sleep or is associated with either an oxygen desaturation of $>3 \%$ or an arousal [3]. The baseline is defined as the mean amplitude of stable breathing and oxygenation in the 2 min preceding the onset of the event [3]. With these criteria, 1405 hypopneas were independently identified in the full-night PSG recordings of our 28 patients by a human expert blinded to the Pes signal (scorer A). For the scoring of a central apnea or a central hypopnea, a clear reduction in Pes swings from the baseline, as defined earlier, is required [3]. According to the guideline [3], there is no relative or absolute reduction in Pes during the event that can be used to differentiate between a central and an obstructive event, thus increasing the difficulty for the automatic differentiation. A second human expert (scorer B) reviewed the manually identified prior hypopneas by scorer A and discarded a hypopnea when its Pes signal did not allow a reliable differentiation. Pes signals often show swallowing or coughing artifacts and may suffer from baseline drifts related to body movements impeding the reliable assessment of respiratory effort. These are known issues in Pes recording [18], as they are inherently related to the technique's complexity, invasiveness, and discomfort caused to the patient [6], thus making it difficult to be avoided during the recording phase. Finally, in a second pass, scorer B manually differentiated the remaining hypopneas into obstructive and central hypopneas using the Pes signal. Undifferentiated hypopneas, mixed hypopneas, and apneas were excluded from this study. A total of $477 \mathrm{ob}-$ structive and 292 central hypopneas were manually scored, thus resulting in an overall of 769 manual, gold-standard hypopnea annotations.

Even though Pes measurement is currently the gold-standard technique for assessment of respiratory effort [3], its complexity is reflected by the number of discarded hypopneas due to Pes artifacts, especially in patients with high intolerance to the Pes catheter. This reinforces the necessity for a noninvasive alternative for hypopnea differentiation. Nonetheless, the final number of available hypopnea annotations in this study represents a distinctive increase in manual, gold-standard (Pes) hypopnea annotations in comparison to other studies that are only analyzed, e.g., an overall of 167 manually scored apneas and hypopneas [10] or 200 apneas [5], respectively, and should allow showing the viability of the proposed automatic methodology.

\section{Preprocessing and Detection of Respiratory Cycles}

The time markers of the 769 manually identified hypopneas, indicating the starting time and the duration in seconds of each hypopnea, were imported for the automatic processing, as the automatic detection of hypopneas with classical PSG criteria [3]

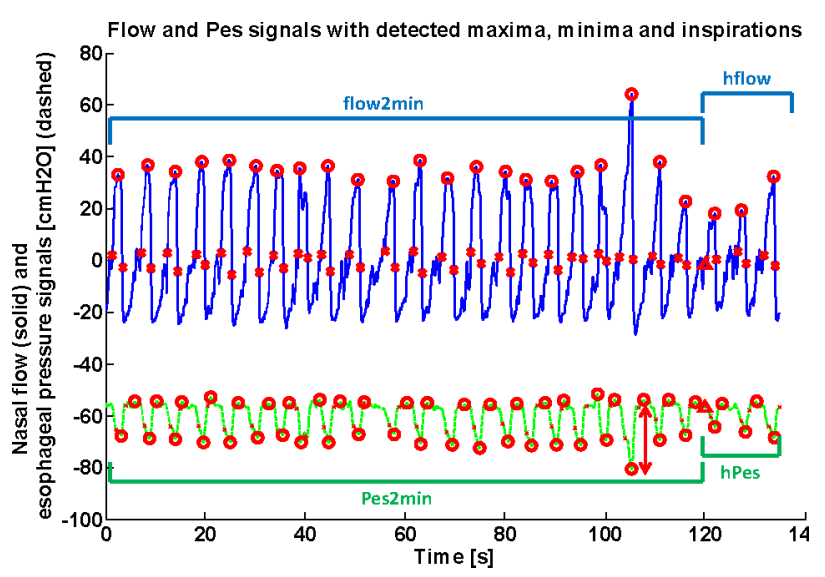

Fig. 1. Example of preprocessing for a central hypopnea. Maxima and minima were detected (circles) for each respiratory cycle and the beginning and ending (crosses) of each inspiration. The manual hyopnea marker (triangle) indicates the beginning of the hypopnea and (arrow) the amplitude difference for each respiratory cycle. The signals were separated into a hypopnea segment (hPes and hflow) and the prior $2 \mathrm{~min}$ (Pes $2 \mathrm{~min}$ and flow $2 \mathrm{~min}$ ). Note the decrease in hflow in respect to flow $2 \mathrm{~min}$ and the significant decrease in respiratory drive for hPes in comparison to Pes $2 \mathrm{~min}$ for this central hypopnea.

has already been solved proficiently [20]. The specific purpose of this study is to find new methods for the automatic differentiation of obstructive and central hypopneas.

The Pes and flow signals presented noise and physiological disturbances that had to be reduced. A low-pass filter with a cutoff frequency of $2.5 \mathrm{~Hz}$ is adequate for the analysis of the flattening airflow patterns when high-frequency oscillations (like snoring) are not to be analyzed [17]. Hence, a five-point moving average (MA) low-pass filter with a cutoff frequency of $2.9 \mathrm{~Hz}$ at $-3 \mathrm{~dB}$ was applied to the flow signal. The Pes signal was interpolated to $32 \mathrm{~Hz}$ and an MA low-pass filter with a cutoff frequency of $1 \mathrm{~Hz}$ at $-3 \mathrm{~dB}$ was applied, as its main frequency content should be located below $1 \mathrm{~Hz}$ [21]. The MA filters were applied to both signals in forward and reverse directions in order to achieve zero phase.

Respiratory periods in the extracted airflow and Pes signals were detected, as described in [16], and the maxima and minima were obtained for each respiratory cycle (see Fig. 1). The corresponding flow/Pes inspiration pairs were then separately extracted in order to allow the individual analysis of each inspiration [16]. In the case that less than two inspirations could not be detected in the Pes signal of a hypopnea due to artifacts, the whole hypopnea was discarded. Seven percent of the 769 hypopneas were discarded by the preprocessing algorithm because of artifacts in the Pes signal. Thus, a total of 715 hypopneas were finally used in the automatic processing and analysis.

The manual hypopnea time markers were used to separate the Pes signal of the hypopnea (hPes), the flow signal of the hypopnea (hflow), and the 2 min prior to the hypopnea's start (Pes2min and flow2min, respectively) (see Fig. 1).

\section{E. Automatic Hypopnea Differentiation With Pes}

The accurateness of the differentiation process will primarily depend on how well the extracted features characterize the 


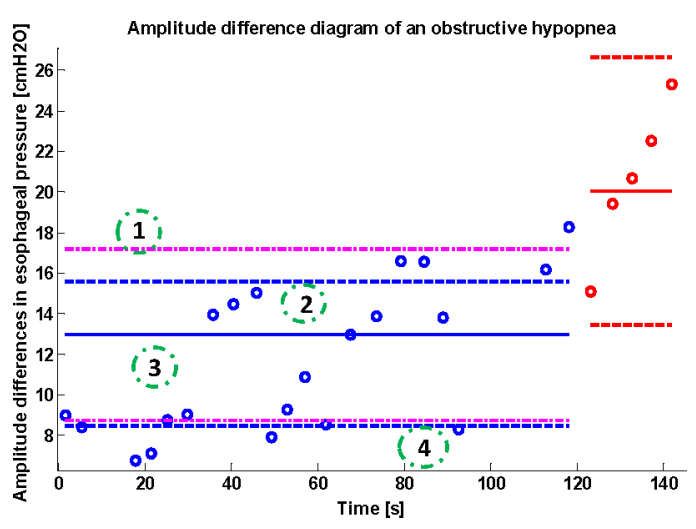

Fig. 2. Example of the distribution of amplitude differences for an obstructive hypopnea. The amplitude difference values (circles) of the hPes segment are located on the right side and its median (solid line on the right) is located above the median (solid line on the left) of Pes2min (circles on the left). This represents an increase in absolute values of respiratory effort. The values of the location index are indicated in the dash-dot circles. Note how the hPes values also show an approximate linear increment, due to the progressive increase in respiratory effort.

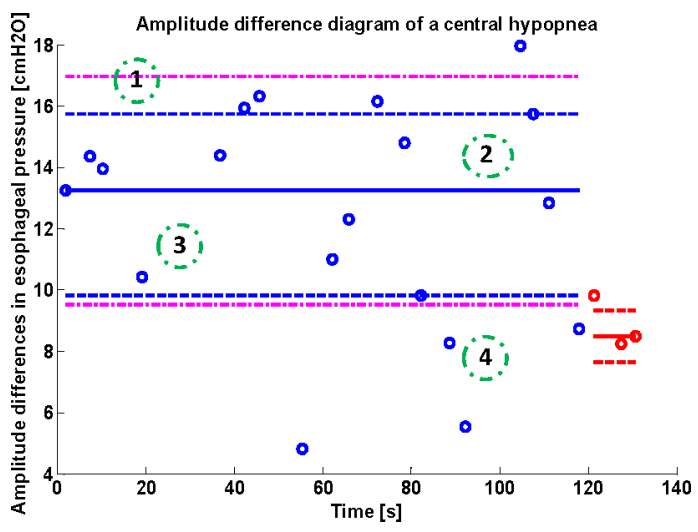

Fig. 3. Example of the distribution of amplitude differences for a central hypopnea. The amplitude difference values (circles) of the hPes segment are located on the right side and its median (solid line on the right) is located below the median (solid line on the left) of Pes2min. This represents a decrease in absolute values of respiratory effort. Note that the hPes difference values for the central apnea do not show a specific pattern.

variation of the pressure swings of central and obstructive hypopneas. As no relative or absolute reduction in Pes during a hypopnea's interval can be used to distinguish between central and obstructive hypopneas [3], the relative changes in amplitude of hPes in respect to Pes2min had to be compared to assess the relative changes in respiratory effort of a hypopnea. In order to avoid the effects of possible baseline drifts during the observed segments of the Pes signal, we were inclined to work with the amplitude difference of the corresponding Pes minimum (inspiration) and Pes maximum (expiration) of each respiratory cycle (see the arrow in Fig. 1). Hence, instead of evaluating the Pes signal's absolute amplitude values, we will be analyzing the relative respiratory effort.

After this preliminary processing, we started searching for the features that could best characterize the differences between obstructive and central hypopneas and could be used to train our classifiers (see Table I). Therefore, the overall number of
TABLE I

FEATURES OF THE AUtOMATIC INVASIVE Classifier

\begin{tabular}{|c|c|c|c|}
\hline \multicolumn{3}{|c|}{ Description } & \multirow{2}{*}{$\begin{array}{c}\text { Description } \\
\text { Median of the amplitude } \\
\text { difference values of hPes + } \\
\text { and its standard deviation, } \\
\text { respectively }\end{array}$} \\
\hline 1 & $\begin{array}{l}\text { Number of amplitude } \\
\text { difference values of hPes }\end{array}$ & 6,7 & \\
\hline 2 & $\begin{array}{l}\text { Median of the amplitude } \\
\text { difference values of } \\
\text { Pes2min }\end{array}$ & 8 & $\begin{array}{l}\text { Relative respiratory } \\
\text { effort index }\end{array}$ \\
\hline 3 & $\begin{array}{l}\text { Median of the superior } \\
\text { group of difference values } \\
\text { of Pes } 2 \mathrm{~min}\end{array}$ & 9 & $\begin{array}{l}\text { Correlation index of the } \\
\text { amplitude difference } \\
\text { values of hPes }\end{array}$ \\
\hline 4 & $\begin{array}{l}\text { Median of the inferior } \\
\text { group of difference values } \\
\text { of Pes } 2 \mathrm{~min}\end{array}$ & 10 & $\begin{array}{c}\text { Median of the } 1^{\text {st }} \\
\text { derivative of the maxima in } \\
\text { hPes }\end{array}$ \\
\hline 5 & $\begin{array}{l}\text { Median of the amplitude } \\
\text { difference values of hPes }\end{array}$ & 11 & $\begin{array}{c}\text { Median of the } 1^{\text {st }} \\
\text { derivative of the minima in } \\
\text { hPes }\end{array}$ \\
\hline
\end{tabular}

amplitude difference values of the hPes signal was used as the first feature (see Table I). As seen in Figs. 2 and 3, we computed the median of the amplitude differences of the Pes2min signal, see solid line on the left in Figs. 2 and 3, dividing the Pes2min amplitude difference values in two groups, one located above this median and another located below. For each of these two groups, their respective median was computed again (see dashed lines on the left in Figs. 2 and 3). The standard deviations (SDs) of the Pes2min signal around the median can also be seen in Figs. 2 and 3 as dash-dot lines on the left. For the hPes signal, the median was computed (see solid line on the right-hand side in Figs. 2 and 3, respectively) \pm the SD of hPes (see dashed red lines on the right-hand side in Figs. 2 and 3, respectively). All these parameters were assembled in the feature pool (see features 2-7 in Table I) for the automatic classifier. With these visual references, we defined a relative respiratory effort index (see feature 8 in Table I), which considered four amplitude difference intervals in which the median of hPes could be located in respect to Pes2min. A numerical value within the range $\{1, \ldots, 4\}$ (see Figs. 2 and 3 ) was assigned to each of these intervals, e.g., if the median of hPes was located above the median of the superior group of Pes2min, then the relative respiratory effort index was assigned the value 1 (see Fig. 2), while if the median of hPes was located below the median of the inferior group of Pes2min, then the relative respiratory effort index was assigned the value 4 (see Fig. 3). Furthermore, we observed that an obstructive hypopnea usually presented a sequential, (approximate) linear increase of amplitude differences (see Fig. 2), representing the progressive increase in respiratory effort typical of obstructive events [3]. The amplitude differences of central hypopneas (see Fig. 3) are usually more constant and do not show a specific recurrent pattern. We assessed this characteristic by computing Pearson's correlation coefficient of the hPes amplitude differences with time. The coefficient's value was then used as a characteristic feature for the differentiation (see feature 9 in Table I). Finally, we also observed a recurrent divergent behavior of the envelope of the $h P e s$ maxima and minima for obstructive hypopneas. Therefore, we computed the median of the first derivatives of the amplitudes of 


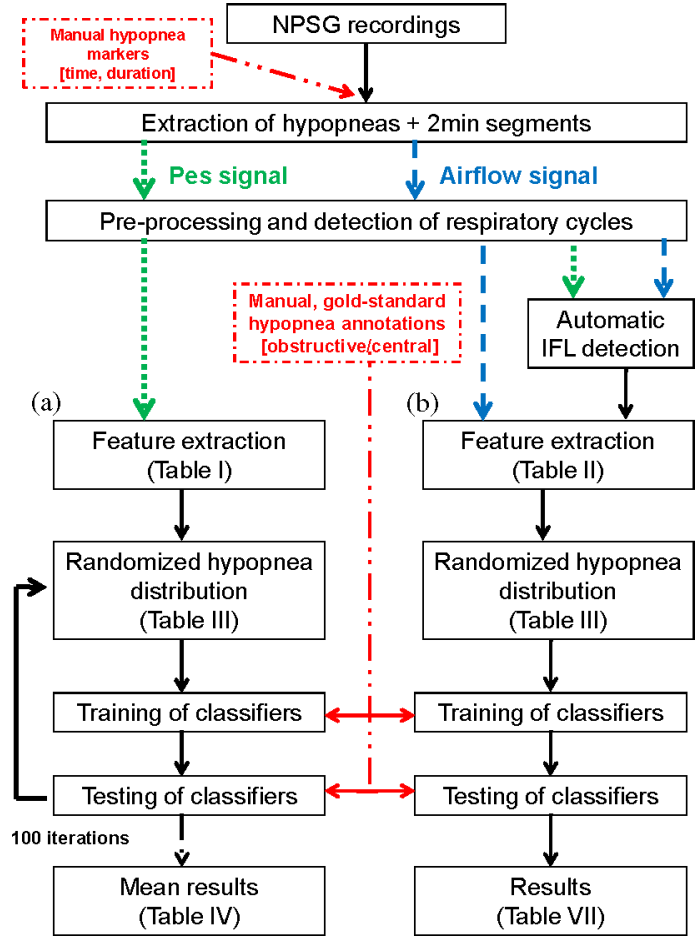

Fig. 4. Flow diagram of the automatic differentiation processes. Both systems shared the signal extraction of the hypopneas and the preprocessing stages. (a) Process of the automatic, invasive differentiation system with the Pes signal (dotted arrows). The 100-fold cross-validation algorithm repeats the distribution/training/testing process to obtain the mean results for 100 iterations (100-fold cross validation). (b) Process of the automatic, noninvasive feasibility study with the airflow (dashed arrows) and Pes signals. IFL are automatically classified with the $\mathrm{P} / V$ relationship. The results represent the hypopnea distribution/training/testing process for a single iteration (cross validation). Both systems scores were validated with the manual, gold-standard hypopnea annotations (dash-dot arrow). The automatic selection process of the optimal feature vector with the forward-selection method and the parameter optimization of the classifiers are not displayed in this diagram. However, this was performed following the same methodology only substituting the test set with the validation set.

the maxima and minima, respectively (see features 10 and 11 in Table I). A step-by-step flow diagram of the automatic invasive classification process is displayed in Fig. 4(a).

\section{F. Feasibility of an Automatic Noninvasive Hypopnea Differentiation System}

As a result of the partial collapse of the UA during sleep, UA resistance increases, usually leading to IFL. IFL is defined as a lack of increase in airflow despite increasing respiratory effort (decreasing intrathoracic pressure) and presents a characteristic inspiratory airflow pattern known as flattening [13]-[17] [see Fig. 5(a)]. Thus, the presence of IFL in form of flattening patterns in the airflow signal provides noninvasive information on changes in UA resistance and respiratory effort [14]-[16].

In an obstructive hypopnea, the decrease in airflow is precisely caused by the commented partial collapse of the UA that leads to an increment in UA resistance, thus preventing the increase in airflow despite the progressively augmenting respiratory effort [1], [3], [4] [see Fig. 5(a)]. In a central hypopnea,
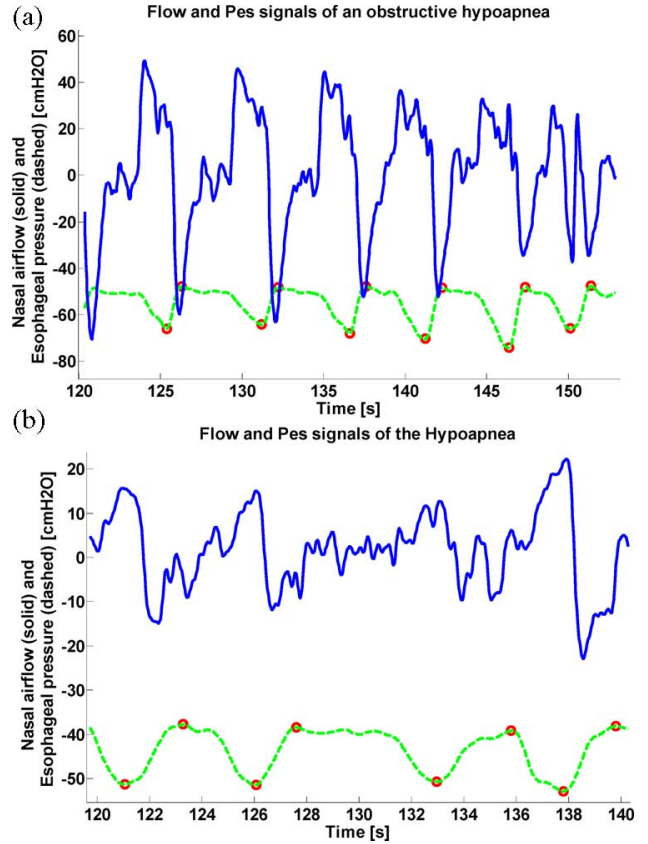

Fig. 5. Examples of the extracted flow and Pes signals for (a) obstructive hypopnea: the inspirations of the obstructive hypopnea show a high incidence of flattening patterns in the airflow signal due to IFL. Also, observe how respiratory effort (Pes signal) progressively increases. (b) Central hypopnea: the presence of IFL in the airflow signal is low, while respiratory effort (Pes signal) does not show significant variations.

however, the decrement of flow is not caused by the collapse of the UA, but because of diminished or even absent respiratory effort due to a lack of neural input from the central nervous system to the diaphragm [1]-[3] [see Fig. 5(b)]. Hence, we hypothesized that the incidence of IFL in the inspiratory cycles of a hypopnea should provide information on the hypopnea's etiology, as we would expect to find more inspirations affected by IFL in an obstructive hypopnea than in a central hypopnea. In order to prove this hypothesis, we needed to objectively quantify and compare the presence of IFL in the inspirations of the obstructive and central hypopneas. Previous studies [15], [16] have already introduced an automatic system that permits an objective assessment of the presence of IFL in inspiratory cycles by means of the gold-standard $P / \dot{V}$ relationship of an inspiration. IFL has been formally defined as a minimum decrease of $1 \mathrm{cmH}_{2} \mathrm{O}(0.7356 \mathrm{mmHg})$ of intrathoracic pressure without a corresponding increase in airway flow rate [13]-[16]. As the corresponding airflow and Pes inspirations of each breath were separately available here after the preprocessing stage, we were able to reconstruct the $P / \dot{V}$ relationship for each inspiration [16] and objectively and automatically assess the presence of IFL [15], [16]. If an inspiration was assessed with IFL, it was assigned the value $\{+1\}$, while if it was a non-IFL inspiration, it obtained the value $\{0\}$.

After this analysis, we sought after the features that could best characterize the differences between obstructive and central hypopneas and could be used with our classifiers (see Table II). Therefore, the mean IFL value for all inspirations of a hypopnea (see feature 1 in Table II) and all inspirations of the 2 min prior 
TABLE II

FEATURes of THE Automatic NoninVAsive Classifier

\begin{tabular}{|c|c|c|c|}
\hline \multicolumn{3}{|c|}{ Description } & Description \\
\hline 1 & $\begin{array}{l}\text { Mean IFL value of all the } \\
\text { inspirations in hflow }\end{array}$ & 6 & $\begin{array}{l}\text { Number of inspirations in } \\
\text { flow } 2 \text { min }\end{array}$ \\
\hline 2 & $\begin{array}{l}\text { Mean IFL value of all the } \\
\text { inspirations in flow } 2 \mathrm{~min}\end{array}$ & 7 & $\begin{array}{l}\text { Mean value of the maximas of all } \\
\text { inspirations in hflow }\end{array}$ \\
\hline 3 & $\begin{array}{l}\text { Number of IFL } \\
\text { inspirations in hflow }\end{array}$ & 8 & $\begin{array}{c}\text { Mean value of the maximas of all } \\
\text { inspirations in flow } 2 \mathrm{~min}\end{array}$ \\
\hline 4 & $\begin{array}{c}\text { Number of IFL } \\
\text { inspirations in flow } 2 \text { min }\end{array}$ & 9 & $\begin{array}{c}\text { Difference between feature } 8 \text { and } \\
\text { feature } 7\end{array}$ \\
\hline 5 & $\begin{array}{l}\text { Number of inspirations in } \\
\text { hflow }\end{array}$ & 10 & $\begin{array}{l}\text { Maximal area of an inspiration in } \\
\text { hflow divided by the maximal } \\
\text { area of an inspiration in flow } 2 \mathrm{~min}\end{array}$ \\
\hline
\end{tabular}

to a hypopnea's onset (see feature 2 in Table II) were computed. We also used the IFL information to reflect the number of inspirations that were affected by IFL in a hypopnea's interval (feature 3) and the number of IFL inspirations in a hypopnea's corresponding 2 min segment (feature 4). A step-by-step flow diagram of the automatic noninvasive classification process is displayed in Fig. 4(b).

The remaining features of our automatic hypopnea differentiation system (see features 5-10 in Table II) were noninvasively obtained solely with the airflow signal. Features $1-4$, however, were obtained with the airflow and the invasive Pes signals $(P / \dot{V}$ relationship). Nevertheless, recent studies [13], [16] have shown that IFL episodes can be assessed noninvasively by just using the airflow signal, and it should be possible to perform the automatic IFL detection without the Pes signal [see Fig. 4(b)]. Therefore, if features 1-4 prove to be relevant for the automatic differentiation of obstructive and central hypopneas, then the development of an entirely noninvasive, automatic classifier based upon the airflow signal should be feasible.

In order to evaluate the discriminatory relevance of features 1 and 2 for the automatic differentiation process of hypopneas, we used the nonparametric Mann-Whitney $U$-test to prove our hypothesis that the IFL values of obstructive hypopneas were significantly $(p<0.01)$ different than those of central hypopneas for features 1 and 2, respectively. Also, the overall mean IFL values for all hypopneas and for all their respective $2 \mathrm{~min}$ segments were separately calculated.

To our knowledge, no other automatic noninvasive system using the airflow signal for the automatic differentiation of central and obstructive hypopneas, which has been validated with an extensive set of gold-standard Pes annotations, has been reported. Thus, there is no immediate reference that we could use to compare the results of our automatic classifier. However, researchers at our partner clinic have developed, independently from this study, a noninvasive hypopnea classification algorithm for human experts for the manual differentiation of hypopneas with standard NPSG signals [22], while the Pes signal was not employed. This classification method consists of a decision tree algorithm that uses the presence of IFL/flattening and contextbased information around a hypopnea for its differentiation. Like the automatic classifiers, the classification results of the manual, noninvasive classification were also validated with the
TABLE III

DISTRIBUTION OF HYPOPNEAS

\begin{tabular}{cccc}
\hline \hline & Obstructive & Central & Total \\
\hline Training set & 157 & 93 & $250(\mathbf{3 5 \%})$ \\
Validation set & 68 & 40 & $108(\mathbf{1 5 \%})$ \\
Test set & 225 & 132 & $357(\mathbf{5 0 \%})$ \\
\hline Total & $\mathbf{4 5 0}$ & $\mathbf{2 6 5}$ & $\mathbf{7 1 5}(100 \%)$ \\
\hline
\end{tabular}

manual, gold-standard (Pes) hypopnea annotations. Therefore, we considered that this manual algorithm could be helpful for evaluating the performance of our automatic classification system. For this purpose, the same gold-standard hypopnea test set was used for the evaluation of both the noninvasive manual and automatic classifiers.

\section{G. Training and Testing of the Automatic Invasive and Noninvasive Classifiers}

Supervised machine-learning techniques, like discriminant analysis (DA) [23], support vector machines (SVMs) [24], and boosting algorithms like adaboost [25], try to predict from the training data to which class each case belongs. During the training phase, the classifiers learn how to solve the problem with a number of input values (features) that should be selected in order to best characterize the function to be predicted by the classifier. The training set is usually composed of a $k$ number of cases (here $k=715$ hypopneas) characterized each by a $n$ number of features (see Tables I and II), thus resulting in a ( $n$-times $k$ )-input matrix, and an output vector of length $k$ containing the desired output classes $\{+1 ;-1\}$ for each case. Here, the desired output values correspond to the manual, gold-standard (Pes) annotations of the obstructive $\{+1\}$ and central $\{-1\}$ hypopneas. Thus, all automatic classifiers in this study were trained and tested with the manual, gold-standard (Pes) annotations. A separate validation set is usually employed in order to fine-tune each classifier's parameters. Then, the optimized and trained classifier is finally run on a test set. The classifier's predicted values are compared to the desired output values in order to estimate the classifier's performance.

The extracted features (see Tables I and II) did not always allow a strict characterization of obstructive and central events individually or collectively. Thus, the best differentiation results can only be usually obtained by using a specific combination of features for each classifier, respectively. Sequential forward-feature selection was used to automatically select the most relevant subset of features for each classifier and achieve the best-possible hypopnea classification results.

A cross-validation algorithm was used in order to randomly assign the hypopneas in a previously designated proportion to the training, test, and validation sets (see Table III and Fig. 4). In order to find the automatic invasive classifier that is closest to generalization, an $i$-fold hold-out cross validation was performed with $i=100$ iterations. The advantage of hold-out cross validation over $i$-fold cross validation is that the proportion of the training/validation split is not dependent on the number 
of folds (iterations). Thus, a $i$ number of random training, validation, and test sets were iteratively created to train, validate, and test our classifiers. Finally, the mean outcome for the classification results of the $i$ iterations was computed to obtain the overall score of the global classifier [see Fig. 4(a)].

Existing supervised machine-learning techniques differ in their generalization capability and computational complexity. For this study, we selected three of the most popular classification techniques with increasing computational complexity.

Five different functions were used during the DA analysis: linear DA (LDA), diagonal linear DA (DLDA), quadratic DA (QDA), diagonal quadratic DA (DQDA), and Mahalanobis DA (MDA). The best-performing function was chosen during the optimization phase with the validation set.

SVM classification (SVM-light v. 6.01, University of Dortmund, Dortmund, Germany) also requires the optimization of several parameters. We used the validation set in order to chose between a polynomial kernel of degrees $(4,8,16,24)$ and a Gaussian kernel with sigma values $(0.1,0.2, \ldots, 0.9)$.

Adaboost combines and weighs a set of weak classifiers to boost them into a strong final classifier [25]. The weak classifiers here were classification and regression trees (CARTs) with an arbitrary number of splits. Different variations of the adaboost algorithm with different generalization properties have also been recently proposed. Standard adaboost [25], "gentle" adaboost [26] and "modest" adaboost [27] are the most important algorithms that will be used here (GML adaboost MATLAB Toolbox, Moscow, Russia). Again, the validation set was used to optimize the commented parameters, such as the number of CART splits $(1,3,6,12,24,36,64$, and 128) and maximum cycles of the adaboost algorithm (100 and 300 iterations).

\section{RESULTS}

\section{A. Results of the Automatic Invasive Hypopnea Classifier}

The invasive classifiers used the features extracted exclusively from the Pes signal in order to differentiate between central and obstructive events. After a 100-fold hold-out cross validation, the best mean classification results for DA were achieved on the validation sets with the DQDA function and a feature vector comprising the features $(1,3,7$, and 8$)$, see Table I. The best classification results for the SVM classifier were achieved with a Gaussian kernel, a sigma of 0.3 and a feature vector with the features $(8,9$, and 11). Of all tested adaboost classifiers, the best results were achieved with the gentle adaboost algorithm, one CART split, and after 100 cycles of maximum iteration. The feature vector for the adaboost classifier comprised the features (1, 2, 4, 5, and 8), see Table I.

The mean classification results of the automatic classifiers in comparison to the manual, gold-standard hypopnea scorings after a 100-fold hold-out cross validation can be seen in Table IV. In order to better observe and compare the classification performance, the results of each classifier for a random test and training set (see Table V) were plotted in form of ROC curves (see Fig. 6). According to the ROC curves, the adaboost classifier seems to deliver a better overall performance than the SVM and the DQDA classifiers.
TABLE IV

MEAN Classification RESUlts FOR A 100-FOLd Hold-Out CROSS VALIDATION

\begin{tabular}{cccc}
\hline \hline & DQDA & SVM & Adaboost \\
\hline Sensitivity & 0.85 & 0.82 & 0.90 \\
Specificity & 0.81 & 0.89 & 0.90 \\
PPV & 0.83 & 0.90 & 0.91 \\
NPV & 0.83 & 0.82 & 0.90 \\
Accuracy & 0.83 & 0.86 & 0.90 \\
Selected features & $1,3,7,8$ & $8,9,11$ & $1,2,4,5,8$ \\
(see Table I) & & & 5 \\
Vector length & 4 & 3 & \\
\hline The labels were defined as $\{+1\}$ for obstructive and $\{-1\}$ for central \\
hypopneas.
\end{tabular}

TABLE V

CLASSIFICATION RESUlTS FOR A RANDOMLY GENERATED TEST AND TRAINING SET

\begin{tabular}{cccc}
\hline \hline & DQDA & SVM & Adaboost \\
\hline Sensitivity & 0.88 & 0.82 & 0.87 \\
Specificity & 0.80 & 0.92 & 0.92 \\
PPV & 0.83 & 0.92 & 0.92 \\
NPV & 0.86 & 0.82 & 0.87 \\
Accuracy & 0.84 & 0.87 & 0.89 \\
\hline
\end{tabular}

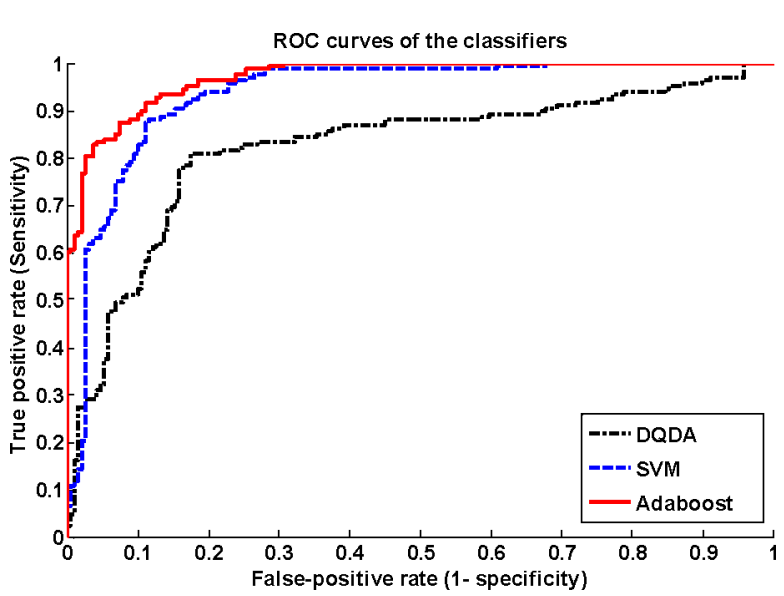

Fig. 6. Receiver-operator characteristic (ROC) curves for the DQDA, the best SVM, and best adaboost classifiers differentiating between central and obstructive hypopneas for a random test and training set. The curves were obtained by varying the threshold value of the classifier's output in the range between -1 and +1 .

\section{B. Results of the Feasibility Study for an Automatic Noninvasive Classifier}

For the automatic noninvasive feasibility study, the mean IFL values $\pm \mathrm{SD}$ for features 1 and 2 (see Table II) for the 715 hypopneas separated into obstructive and central hypopneas can be seen in Table VI. The mean IFL values were significantly different $(p<0.01)$ between obstructive and central hypopneas for the inspirations during a hypopnea (see feature 1 in Table II) and for the inspirations in the 2 min. prior to a hypopnea's onset (see feature 2 in Table II). The difference between the IFL mean values between central and obstructive hypopneas was significantly higher for feature 1 , see Table VI. 
TABLE VI

MEAN IFL VALUES \pm SD FOR THE INSPIRATIONS IN A HYPOPNEA AND THE PRECEDING 2 MIN SEGMENT

\begin{tabular}{ccc}
\hline \hline & $\begin{array}{c}\text { Mean } \text { 土 SD IFL values } \\
\text { for hypopneas (feature 1) }\end{array}$ & $\begin{array}{c}\text { Mean } \pm \text { SD IFL values } \\
\text { for the 2 min preceding } \\
\text { a hypopnea (feature 2) }\end{array}$ \\
\hline $\begin{array}{c}\text { Obstructive } \\
\text { hypopneas } \\
\text { Central } \\
\text { hypopneas }\end{array}$ & $0.47 \pm 0.34$ & $0.39 \pm 0.24$ \\
\hline
\end{tabular}

Significant differences $(p<0.01)$ were found between the IFL values for central and obstructive hypopneas for both features.

TABLE VII

ClASSIFICATION RESUlTS FOR THE AUTOMATIC CLASSIFIER OF THE NONINVASIVE FEASIBILITY STUDY WITH A RANDOMLY GENERATED TEST AND TRAINING SET

\begin{tabular}{ccc}
\hline \hline & $\begin{array}{c}\text { Automatic } \\
\text { classification }\end{array}$ & $\begin{array}{c}\text { Manual } \\
\text { classification }\end{array}$ \\
\hline Sensitivity & 0.72 & 0.68 \\
Specificity & 0.71 & 0.88 \\
PPV & 0.81 & 0.91 \\
NPV & 0.60 & 0.62 \\
Accuracy & $\mathbf{0 . 7 2}$ & $\mathbf{0 . 7 5}$ \\
Features & $1,3,9,10$ & Flattening and context- \\
& (see Table II) & based information \\
\hline
\end{tabular}

The same hypopnea test set was used for the manual and the automatic classifiers. The labels were defined as $\{+1\}$ for obstructive and $\{-1\}$ for central hypopneas.

The best results for the automatic classification were achieved with a DQDA classifier and features (1, 3, 9, and 10), see Table VII. The automatic classifier delivered a similar accuracy, see Table VII, as the manual classifier on the same gold-standard annotation test set (see Table III).

\section{DISCUSSION AND CONCLUSION}

This paper has presented an invasive and a noninvasive method for the automatic differentiation of obstructive and central hypopneas. An overall of 715 manual, gold-standard hypopnea scorings of 28 patients were automatically classified, thus representing a significant increase in the number of patients with systematic Pes measurement and in the number of manual, gold-standard annotations than in comparable studies [5], [7], [10].

In the first part of this paper, the viability of an automatic, invasive classifier for the differentiation of obstructive and central hypopneas with the Pes signal has been shown. As the Pes differentiation criteria of hypopneas are identical to those of apneas [3], the presented automatic differentiation system should also be applicable for the automatic differentiation of central and obstructive apneas. The obtained results only varied slightly when a low-pass MA filter with a higher cutoff frequency (e.g., $2.9 \mathrm{~Hz}$ ) was applied to the Pes-signal, underlining the robustness of the presented methodology. Still, the best overall results here were achieved with the described $1 \mathrm{~Hz}$ low-pass MA filter. However, for some Pes inspirations, this filter may possibly suppress some high-frequency components. This can in most part be avoided using the $2.9 \mathrm{~Hz}$ filter instead. Therefore, for future applications, the cutoff frequency of the Pes signal's filter should be carefully selected.

The information contained in the features extracted from the Pes signal seems to be adequate for the automatic differentiation process. It is remarkable that feature 8 (see Table I) was the only feature that was automatically selected by the forward-selection method for all three classifiers, underlining the importance of the relative respiratory effort index for the differentiation process. The adaboost classifier showed the best overall classification results (see Table IV, Fig. 6), although it was also the classifier with the highest computational complexity, the largest feature vector, and processing time. The DQDA classifier showed a remarkable overall performance given the fact that it is the classifier with the lowest computational complexity (see Fig. 6). As the classifiers were trained and validated with manual, gold-standard (Pes) annotations, the validity of the automatic classifier's scorings should consequently be ensured. The elevated accuracy of the automatic invasive hypopnea classifier after a 100 -fold hold-out cross validation, see Table IV, and using only $35 \%$ of the hypopneas for the training set, see Table III, underlines the system's robustness and generalization capability. However, in this study, only one human scorer manually differentiated the hypopneas with the Pes signal. This limits the generalization of the automatic classification scores, as human scorings usually suffer from subjective interpretation and human interscorer agreement usually ranges between $80 \%$ and $90 \%$ [8], [13]. Nevertheless, the manual annotations that have been employed here have permitted showing the technical viability of the presented automatic, invasive hypopnea differentiation methodology, given the promising automatic scoring results. However, an additional and extensive test set of manual, gold-standard (Pes) annotations that have been independently and blindly scored by at least two human experts, will be necessary before a prospective clinical validation of this automatic invasive classifier is achieved.

In the second part of this paper, the feasibility of a new noninvasive approach for the differentiation of hypopneas has been shown. Unlike the invasive classifier which, as commented earlier, should be applicable to both apneas and hypopneas, the noninvasive classifier should solely work with hypopneas, as it would be difficult to assess flattening patterns during apneas, whose airflow signal is very close to the baseline. Features 1 and 3 (see Table II) were automatically selected by the forward-selection method for the automatic, noninvasive hypopnea differentiation. This underlines the importance of the information contained in the flattening airflow patterns of IFL episodes for the differentiation process. We have, to our knowledge for the first time, objectively demonstrated that obstructive hypopneas have a significantly higher $(p<0.01)$ incidence of IFL episodes than central hypopneas (see Table VI). For the automatic, objective assessment of IFL, we used the gold-standard $P / \dot{V}$ relationship that has been extensively described in previous studies [14]-[16] and is considered one of the most objective and accurate methods to assess IFL [14]-[16]. Furthermore, we have also shown that the difference between the overall mean IFL scores of central and obstructive hypopneas, when observing just the hypopnea episode (0.13), are higher than during 
the corresponding 2 min segment prior to the hypopnea's onset (0.08) (see Table VI). This should be the reason why the automatic forward-selection method priorized feature 1 over feature 2 for the feature vector of the automatic classifier (see Table VII). It should also be pointed out that the 2 min segments of obstructive hypopneas presented a significantly $(p<0.01)$ higher IFL incidence than the 2 min segments of central hypopneas. Thus, it appears that an obstruction seems to announce itself even before the onset of a hypopnea.

Even though features 8 and 10 (see Table II) of the automatic hypopnea classifier were obtained noninvasively just with the airflow signal, features 1 and 3 (see Table II) containing the IFL-related information were obtained by means of the invasive $P / \dot{V}$ relationship [16] [see Fig. 4(b)]. However, recent developments [13], [16] have suggested new approaches that allow an objective and accurate noninvasive assessment of IFL episodes only with the airflow signal. Hence, these methods [13], [16] should permit to obtain features 1 and 3 noninvasively without the Pes signal, and consequently, allow the development of an entirely noninvasive automatic hypopnea differentiation system based upon the airflow signal [see Fig. 4(b)].

Other noninvasive systems in the literature [7]-[10] presented a significantly smaller amount of analyzed hypopneas than our system, did not use gold-standard Pes signal annotations to validate their classifier, and/or analyzed apneas instead of hypopneas, thus increasing the difficulty to fairly compare these studies' respective classification results with those of our automatic system. This is why we used a manual noninvasive classification algorithm [22] to evaluate our automatic classifiers' performance. This manual noninvasive classification by human experts was only based upon features extracted from standard NPSG signals (see Table VII), as the Pes signal was not employed for the classification. In the same way as with the automatic, invasive classifier, also here the manual, goldstandard (Pes) hypopnea annotations, were employed for the validation of the noninvasive manual classification algorithm and the training and testing of the automatic classifier. Therefore, the validity of the differentiation scores should here again be ensured.

The results for our automatic hypopnea classifier seem promising, as the automatic classifier's accuracy is approximately on par with the accuracy obtained with the manual, noninvasive scoring (see Table VII). Like most systems relying only on NPSG signals [5], the manual classification algorithm showed a strong tendency to over classify central events $(0.88)$ in detriment of the correct identification of obstructive events (0.68) (see Table VII). The automatic classifier, however, showed a better balanced identification performance of the two hypopnea classes (see Table VII). This underlines the importance of using objectively extracted respiratory-effort-related information (IFL/flattening) during the hypopnea's differentiation process. However, further development of the automatic, noninvasive differentiation system will be necessary to obtain a similar robustness, classification, and generalization performance comparable to that of the invasive automatic classifier (see Table IV). Still, we are confident that the promising insights obtained in this study have shown the feasibility and could facil- itate the implementation of an automatic, entirely noninvasive hypopnea differentiation system solely based upon the airflow signal. This new system could then be validated with an extensive set of manually or automatically created gold-standard (Pes) hypopnea annotations.

\section{ACKNOWLEDGMENT}

C. Morgenstern would like to thank W. Galetke, M. Treml, N. Anduleit, and K. Richter of the Zentrum für Schlaf- und Beatmungsmedizin, Krankenhaus Bethanien, Solingen, Germany and A. Oltmann, M. Saka, D. Sommermeyer, and B. Schöller of MCC GmbH \& Co. KG, Karlsruhe, Germany for their assistance and support during the development of this study.

\section{REFERENCES}

[1] A. I. Pack, "Advances in sleep disordered breathing," Amer. J. Respir. Crit. Care Med., vol. 172, pp. 7-15, 2006.

[2] C. Guilleminault and A. Robinson, "Central sleep apnea, upper airway resistance and sleep," Sleep Med., vol. 7, pp. 189-191, 2006.

[3] American Academy of Sleep Medicine Task Force. Sleep-Related Breathing Disorders in Adults: Recommendations for Syndrome Definition and Measurement Techniques in Clinical Research. The Report of an American Academy of Sleep Medicine Task Force, Sleep, vol. 22, no. 5, pp. 667-689, 1999.

[4] C. Iber, S. Anoni-Israel, A. L. Chesson, and S. F. Qua, The AASM Manual for the Scoring of Sleep and Associated Events. Amer. Acad. Sleep Med., Westchester, IL, 2007.

[5] Y. M. Luo, J. Tang, J. Steier, N. S. Zhong, J. Moxham, and M. I. Polkey, "Distinguishing obstructive from central sleep apnea events: Diaphragm electromyogram and esophageal pressure compared," Chest, vol. 135, no. 5, pp. 1133-1141, 2009.

[6] R. Chervin and M. Aldrich, "Effects of esophageal pressure monitoring on sleep architecture," Amer. J. Respir. Crit. Care Med., vol. 156, pp. 881885, 1997.

[7] O. Fontenla-Romero, B. Guijarro-Berdiñas, A. Alonso-Betanzos, and V. Moret-Bonillo, "A new method for sleep apnea classification using wavelets and feedforward neural networks," Artif. Intell. Med., vol. 34, no. 1 , pp. 65-76, 2005.

[8] H. Steltner, R. Staats, J. Timmer, M. Vogel, J. Guttmann, H. Matthys, and J. C. Virchow, "Diagnosis of sleep apnea by automatic analysis of nasal pressure and forced oscillation impedance," Amer. J. Respir. Crit. Care Med., vol. 165, pp. 940-944, 2002.

[9] F. C. Yen, K. Behbehani, E. A. Lucas, J. R. Burk, and J. R. Axe, "A nonnvasive technique for detecting obstructive and central sleep apnea," IEEE Trans. Biomed. Eng., vol. 44, no. 12, pp. 1262-1268, Dec. 1997.

[10] J. Argod, J. L. Pépin, and P. Lévy, "Differentiating obstructive and central sleep respiratory events through pulse transit time," Amer. J. Respir. Crit. Care Med., vol. 158, pp. 1778-1783, 1998.

[11] C. Morgenstern, M. Schwaibold, W. Randerath, A. Bolz, and R. Jané "Automatic differentiation of obstructive and central hypopneas with esophageal pressure measurement during sleep," in Proc. IEEE Eng. Med. Biol. Soc. 31st Annu. Int. Conf., Minneapolis, MN, 2009, pp. 7102-7105.

[12] G. A. Gould, K. F. Whyte, G. B. Rhind, M. A. Airlie, J. R. Catterall, C. M. Shapiro, and N. J. Douglas, "The sleep hypopnea syndrome," Amer. Rev. Respir. Dis., vol. 137, pp. 895-898, 1988.

[13] I. Ayappa, R. G. Norman, A. C. Krieger, A. Rosen, R. L. O’Malley, and D. M. Rapoport, "Non-invasive detection of respiratory effort-related arousals (RERAs) by a nasal cannula/pressure transducer system," Sleep, vol. 23, no. 6, pp. 763-771, 2000.

[14] J. Hosselet, R. Norman, A. Ayappa, and D. Rapoport, "Detection of flow limitation with a nasal cannula/ pressure transducer system," Amer. J. Respir. Crit Care Med., vol. 157, pp. 1461-1467, 1998.

[15] S. A. Clark, C. R. Wilson, M. Sato, D. Pegelow, and J. A. Dempsey, "Assessment of inspiratory flow limitation invasively and noninvasively during sleep," Amer. J. Respir. Crit. Med., vol. 158, pp. 713-722, 1998.

[16] C. Morgenstern, M. Schwaibold, W. Randerath, A. Bolz, and R. Jané, "Assessment of changes in upper airway obstruction by automatic identification of inspiratory flow limitation during sleep," IEEE Trans. Biomed. Eng., vol. 56, no. 8, pp. 2006-2015, Aug. 2009. 
[17] T. Aittokallio, O. Nevalainen, U. Pursiheimo, T. Saaresranta, and O. Polo, "Classification of nasal inspiratory flow shapes by attributed finite automata," Comput. Biomed. Res., vol. 32, pp. 34-55, 1999.

[18] J. O. Benditt, "Esophageal and gastric pressure measurements," Respir. Care, vol. 50, no. 1, pp. 68-75, 2005

[19] C. Kushida, A. Giacomini, M. K. Lee, C. Guilleminault, and W. C. Dement, "Technical protocol for the use of esophageal manometry in the diagnosis of sleep-related breathing disorders," Sleep Med., vol. 3, pp. 163-173, 2002.

[20] P. Varady, T. Micsik, S. Benedek, and Z. Benyo, "A novel method for the detection of apnea and hypopnea events in respiration signals," IEEE Trans. Biomed. Eng., vol. 49, no. 9, pp. 936-942, Sep. 2002.

[21] P. Ask, P. A. Oberq, and L. Tibbling, "Frequency content of esophageal peristaltic pressure," Amer. J. Physiol. Endocrinol. Metab, vol. 236, pp. E296-E300, 1979.

[22] W. Randerath, C. Morgenstern, A. Oltmann, C. Priegnitz, M. Treml, and N. Anduleit, "Evaluation of a new PSG based algorithm to differentiate obstructive and central hypopnoeas," presented at the 20th Annu. Conf. Eur. Respir. Soc., Barcelona, Spain, 2010.

[23] W. J. Krzanowski, Principles of Multivariate Analysis. London, U.K.: Oxford Univ. Press, 1988

[24] V. Vapnik, The Nature of Statistical Learning Theory. New York: Springer-Verlag, 1995.

[25] Y. Freund and R. Schapire, "Game theory, on-line prediction and boosting," in Proc. 9th Annu. Conf. Comp. Learning Theory, 1996, pp. 325-332.

[26] J. Friedman, T. Hastie, and R. Tibshirani, "Additive logistic regression: A statistical view of boosting," Ann. Statist., vol. 38, no. 2, pp. 337-374, 2000.

[27] A. Vezhnevets and V. Vezhnevets, "Modest AdaBoost-teaching AdaBoost to generalize better," presented at the Graphicon 2005, Novosibirsk, Akademgorodok, Russia.

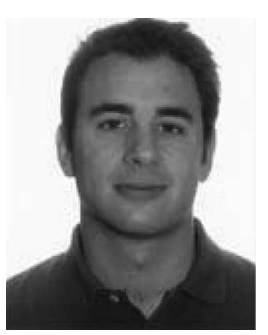

Christian Morgenstern (S'04-M'10) received the E.E.I.T. degree from the University of Karlsruhe, Karlsruhe, Germany, in 2004, and the Ph.D. degree from the Universitat Politècnica de Catalunya, Barcelona, Spain, in February 2010. He is currently working toward the M.D. degree at the University of Barcelona, Barcelona, Spain

In 2003, he was a Visiting Student at the Center for Biological and Computational Learning, Massachusetts Institute of Technology, Cambridge, where he completed the Master's thesis under Prof. T. Poggio and Dr. B. Heisele. His current research interests include signal processing, analysis and modeling of human respiration during sleep, and applications of machine learning methods to solve biomedical problems.

Matthias Schwaibold received the E.E.I.T. degree from the University of Karlsruhe, Karlsruhe, Germany, in 2000.

$\mathrm{He}$ is currently the Head of the Research and Development Department for sleep medicine, MCC GmbH \& Company KG, Karlsruhe, member of the Weinmann group. He has been involved in development and publication of several innovative solutions for sleep staging, sleep and cardiac screening with an extended pulse oximeter, as well as autoCPAP, autoBiLevel, and servoventilation algorithms. His research interests include signal processing, treatment algorithms, and future technological trends in sleep medicine.

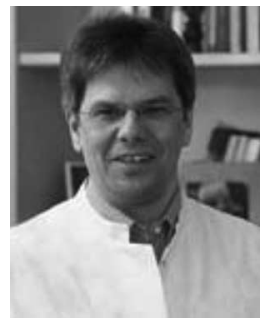

Winfried J. Randerath received the M.D. degree from the University of Düsseldorf, Düsseldorf, Germany, in 1988, and the Ph.D. degree from the University of Witten/Herdecke, Witten, Germany, in 2000.

$\mathrm{He}$ is currently a Professor at the Institute for Pneumology, University of Witten/Herdecke, and Director of the Bethanien Hospital, Solingen, Germany. His research interests include diagnosis and treatment of sleep-related breathing disorders (obstructive sleep apnea, Cheyne-Stokes respiration, obesityhypoventilation), capnometry, and respiratory failure.

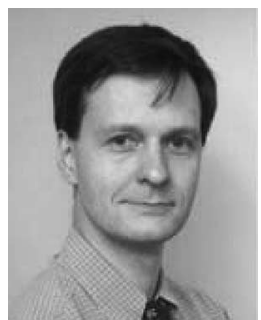

Armin Bolz received the Ph.D. degree in physics from Friedrich-Alexander University, Erlangen, Germany in 1991.

From 1991 to 1999, he was Director of R\&D with Biotronik, Germany, where he was enagaged in research on implantable pacemakers and defibrillators. In 1999, he became a Professor at the Institute for Biomedical Engineering, Karlsruhe Institute of Technology (KIT), Karlsruhe. In 2001, he founded the technology company Corscience $\mathrm{GmbH} \& \mathrm{Com}-$ pany $\mathrm{KG}$, Erlangen. He is the author or coautor of 70 publications and holds many patents. His current research interests include defibrillators, vital signs sensors, biosignal analysis, and telemedicine.

Dr. Bolz was the President of the German Society of Biomedical Engineering (DGBMT) during 1999-2001. In 2001, he was a Member of the Board of the Verband der Elektrotechnik Elektronik Informationstechnik e.V. (VDE). He has received several innovation prizes.

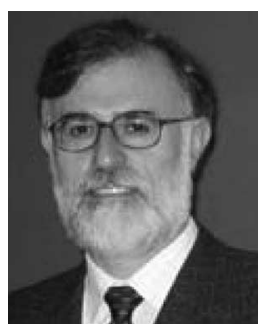

Raimon Jané (M'91) received the Ph.D. degree from the Universitat Politècnica de Catalunya (UPC), Barcelona, Spain, in 1989.

$\mathrm{He}$ is currently the Director of research in the Department of Automatic Control (ESAII), UPC, and the Scientific Group Leader of the Biomedical Signal Processing and Interpretation Group, Institut de Bioenginyeria de Catalunya (IBEC), Barcelona. Since 2008, he has been the Principal Investigator of the Biomedical Signals and Systems (SISBIO) Group, El Centro de Investigación Biomédica en Red (CIBER) de Bioingeniería, Biomateriales y Nanomedicina (CIBER-BBN). He is currently a Professor of the Master's and a Coordinator of the Ph.D. programs on biomedical engineering. His research interests include multimodal and multiscale biomedical signal processing in cardiorespiratory and brain diseases.

Prof. Jané is the Vice President of the Spanish Society of Biomedical Engineering (SEIB). In 2005, he was a recipient of the Barcelona City Prize, of the Barcelona Council, in the area of technology research. 